This item was submitted to Loughborough's Research Repository by the author.

Items in Figshare are protected by copyright, with all rights reserved, unless otherwise indicated.

\title{
Control of an actively constrained robotic joint for passive deployment applications
}

PLEASE CITE THE PUBLISHED VERSION

PUBLISHER

Professional Engineering Publishing / @ IMECHE

VERSION

VoR (Version of Record)

LICENCE

CC BY-NC-ND 4.0

\section{REPOSITORY RECORD}

Reedman, Adam V.C., and Kaddour Bouazza-Marouf. 2019. "Control of an Actively Constrained Robotic Joint for Passive Deployment Applications". figshare. https://hdl.handle.net/2134/5149. 
This item was submitted to Loughborough's Institutional Repository (https://dspace.lboro.ac.uk/) by the author and is made available under the following Creative Commons Licence conditions.

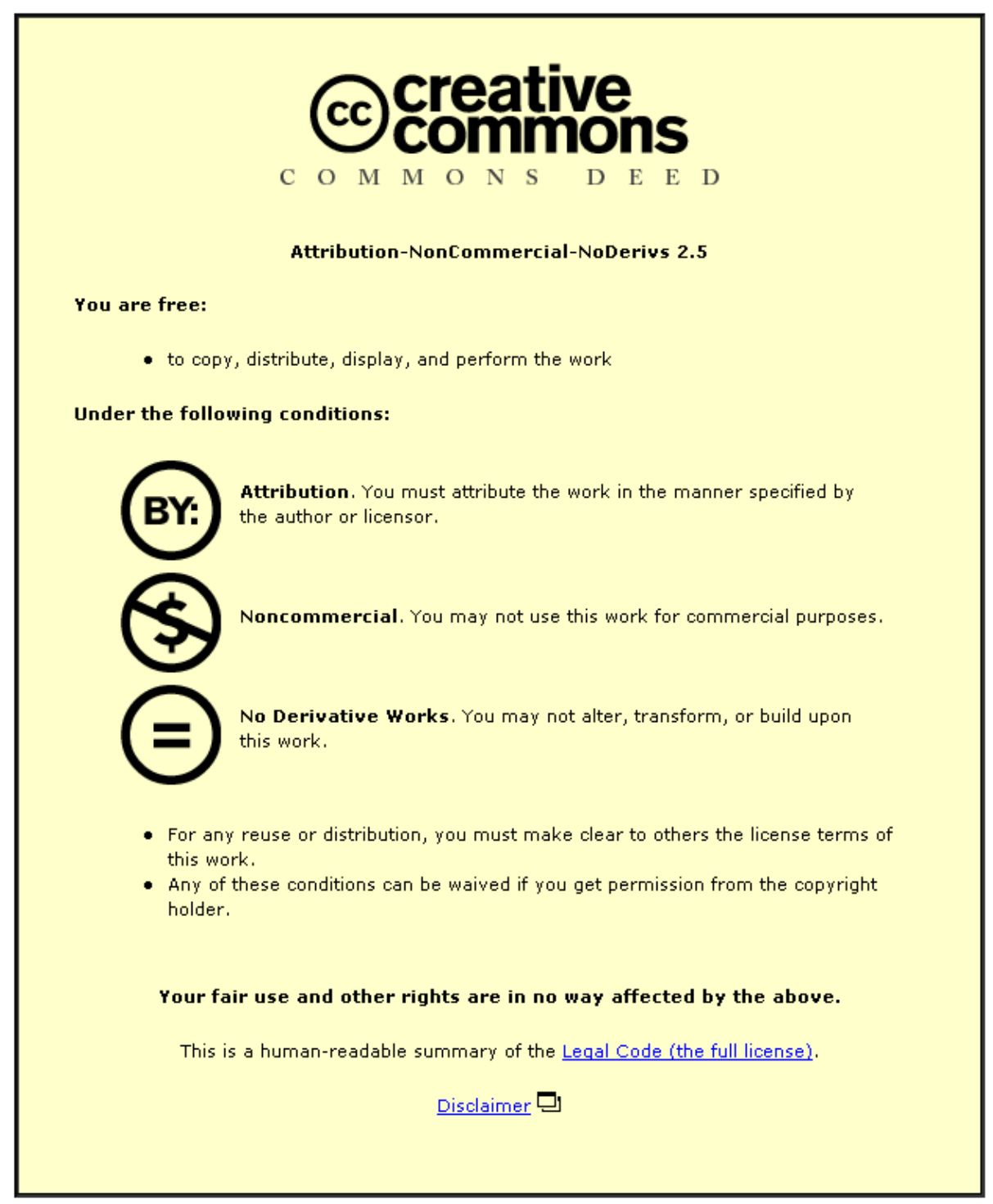

For the full text of this licence, please go to: http://creativecommons.org/licenses/by-nc-nd/2.5/ 


\title{
Control of an actively constrained robotic joint for passive deployment applications
}

\author{
A V C Reedman and K Bouazza-Marouf * \\ Wolfson School of Mechanical and Manufacturing Engineering, Loughborough University, Leicestershire, UK
}

\begin{abstract}
The design and control of an actively constrained revolute joint with backlash cancellation for passively deployed devices is presented in this paper. The drive mechanism consists of two motordriven worms coupled to a single worm wheel. A mathematical model of the system is used in order to develop a backlash cancellation strategy and computed-torque motion control algorithm. Experimental results show that the position of the joint can be successfully controlled to track a trajectory generated from a user-input force command signal while cancelling backlash at the gear interface.
\end{abstract}

Keywords: backlash cancellation, computed-torque control, robotics

\begin{tabular}{|c|c|}
\hline \multicolumn{2}{|c|}{ NOTATION } \\
\hline$C_{\mathrm{f} \alpha}, C_{\mathrm{f} \beta}, C_{\mathrm{f} \theta}$ & $\begin{array}{l}\text { viscous friction coefficient of the } \\
\alpha \text {-worm system, the } \beta \text {-worm system and }\end{array}$ \\
\hline$C_{\alpha}, C_{\beta}, C_{\theta}$ & $\begin{array}{l}\text { the worm-wheel system respectively } \\
\text { equivalent viscous friction coefficient of } \\
\text { the } \alpha \text {-worm system, the } \beta \text {-worm system } \\
\text { and the worm-wheel system } \\
\text { respectively }\end{array}$ \\
\hline$F_{\mathrm{N} \alpha}, F_{\mathrm{N} \beta}$ & $\begin{array}{l}\text { reaction force at the interface between } \\
\text { the } \alpha \text {-worm and the worm wheel and } \\
\text { between the } \beta \text {-worm and the worm } \\
\text { wheel respectively }\end{array}$ \\
\hline$F_{\mathrm{S} \alpha}, F_{\mathrm{S} \beta}$ & $\begin{array}{l}\text { Coulomb friction force between the } \\
\alpha \text {-worm and the worm wheel and } \\
\text { between the } \beta \text {-worm and the worm } \\
\text { wheel respectively }\end{array}$ \\
\hline & applied user-input force \\
\hline$F_{\alpha}, F_{\beta}$ & $\begin{array}{l}\text { equivalent force generated by the torque } \\
\text { from the motors }\end{array}$ \\
\hline$J_{\alpha}, J_{\beta}, J_{\theta}$ & $\begin{array}{l}\text { inertia of the } \alpha \text {-worm and the motor, of } \\
\text { the } \beta \text {-worm and the motor and of the } \\
\text { worm wheel, shaft and robot arm } \\
\text { respectively }\end{array}$ \\
\hline$M_{\alpha}, M_{\beta}, M_{\theta}$ & $\begin{array}{l}\text { equivalent mass of } J_{\alpha}, J_{\beta} \text { and } J_{\theta} \\
\text { respectively }\end{array}$ \\
\hline
\end{tabular}

The MS was received on 13 June 2001 and was accepted after revision for publication on 24 September 2001.

*Corresponding author: Wolfson School of Mechanical and Manufacturing Engineering, Loughborough University, Loughborough, Leicestershire LE11 3TU, UK.

$\begin{array}{ll}r_{\alpha}, r_{\beta}, r_{\theta} & \begin{array}{l}\text { radius of contact of the } \alpha \text {-worm with the } \\ \text { worm wheel, of the } \beta \text {-worm with the }\end{array} \\ & \text { worm wheel and of the } \alpha \text { - and } \beta \text {-worms } \\ & \text { respectively } \\ & \text { equivalent linear displacement of the } \\ & \alpha \text {-worm, } \beta \text {-worm and worm wheel } \\ & \text { respectively }\end{array}$

\section{INTRODUCTION}

Development of robotic devices that interact closely with humans has been of particular interest to the surgical industry for the last 20 years. In an attempt to improve the accuracy and repeatability of surgical procedures, robotic tool-positioning and tool-guiding devices have been introduced into the operating theatre.

Most of the robotic devices that are currently used to aid surgeons in performing operational tasks have been based on modified industrial manipulators. The best known of these modified robots is ROBODOC $[\mathbf{1}, \mathbf{2}]$. ROBODOC was designed for machining a cavity in bone 
to receive a prosthetic component during cementless total hip replacement. The surgeon oversees the operation but has no control over the motion other than the ability to stop and start the robot using a control pendant. Although these large industrial systems are highly accurate, they have typically been designed to meet the requirements of high-speed and high-torque applications. The introduction of a large, powerful robot into an environment such as the operating theatre casts doubt on the safety of the patient, surgeon and other operating room staff [3]. It is for this reason that the makers of ROBODOC, and other similar devices such as the master-slave robot Da Vinci [4], have been required to implement many layers of software and hardware safety systems in order to ensure that unauthorized motion cannot be made. It seems that more attention has been focused on limiting rather than eliminating the ability to do harm.

Custom-built robotic devices, such as ACROBOT (active constraint ROBOT) [5] and PADyC (passive arm with dynamic constraints) [6], have been a more attractive alternative to the larger and more expensive industrial systems. ACROBOT is a four-degree-of-freedom (4-DOF) manipulator designed to help the surgeon perform total knee replacement surgery. The surgeon controls the motions of the manipulator by applying a force to the control handle in order to drive the joints of the robot. All of the joints are backdriveable, and a d.c. motor at each joint controls the resistive force that the surgeon feels using a force control strategy. The workspace of the robot is actively constrained to confine the end-effector to remain within a preplanned safe working region. However, while the ACROBOT system requires physical input from the surgeon in order to move the manipulator, the force-controlled servomotors in each joint are still powerful enough to provide motion against the user. This again raises many issues regarding surgeon and patient safety. Another proposed robot assistant device is PADyC, a robot incapable of motion of its own. At every joint there exists a pair of overrunning clutches, each running on a separate motor driven hub. By controlling the speed of each motor, one clutch limits the maximum allowable speed in the clockwise direction while the second clutch restricts speed in the anticlockwise direction. It was demonstrated that PADyC could be used to confine the motion of the surgeon within a predefined work area and, to a limited extent, follow a predefined path. With $20 \mathrm{~N}$ of force applied on the control handle, the 2-DOF prototype of PADyC exhibited up to $20 \mathrm{~mm}$ of error at the tool tip with link lengths of $0.25 \mathrm{~m}$ [7]. The error was attributed to joint flexibility and backlash in the clutch mechanism. Errors of this magnitude cannot be tolerated in most surgical applications.

In this paper, a robot joint architecture designed to cancel backlash is presented. A conceptual overview of the mechanism is given for a system that has been designed to be inherently fail safe, non-backdriveable, and suitable for use in applications where direct control of the end-effector (surgical tool) by a human is required. A mathematical model of the mechanism is then presented and used to develop a computed-torque control algorithm for regulating position. A brief description of the experimental set-up and control system architecture is also included. Simulation and experimental results of the mechanism and control algorithm are given, followed by concluding remarks.

\section{CONCEPTUAL OVERVIEW}

In gear system design, a small amount of backlash is required in order to allow for thermal expansion, lubrication and lower frictional losses within a drivetrain [8]. In many robotic applications, even minute amounts of backlash can cause errors in position that are not acceptable in demanding applications such as surgery. For example, if a single link of a robot arm exhibits $\pm 0.0087 \mathrm{rad}$ ( or $\pm 0.5^{\circ}$ ) of backlash, there will be an error of $\pm 3.5 \mathrm{~mm}$ at the end of a $0.4 \mathrm{~m}$ link. In order to reduce the backlash to an acceptable level, gear train housings must be manufactured to high engineering tolerances. This is expensive in terms of both the machinery required and the time taken for manufacture. Another solution would be to use antibacklash gears, readily available from many gear manufacturers. However, these antibacklash gears are spring-loaded systems and still allow relative motion between input and output shafts under certain loading conditions. The proposed system, presented in this paper, is a robotic joint designed to cancel backlash.

The joint, shown schematically in Fig. 1, consists of

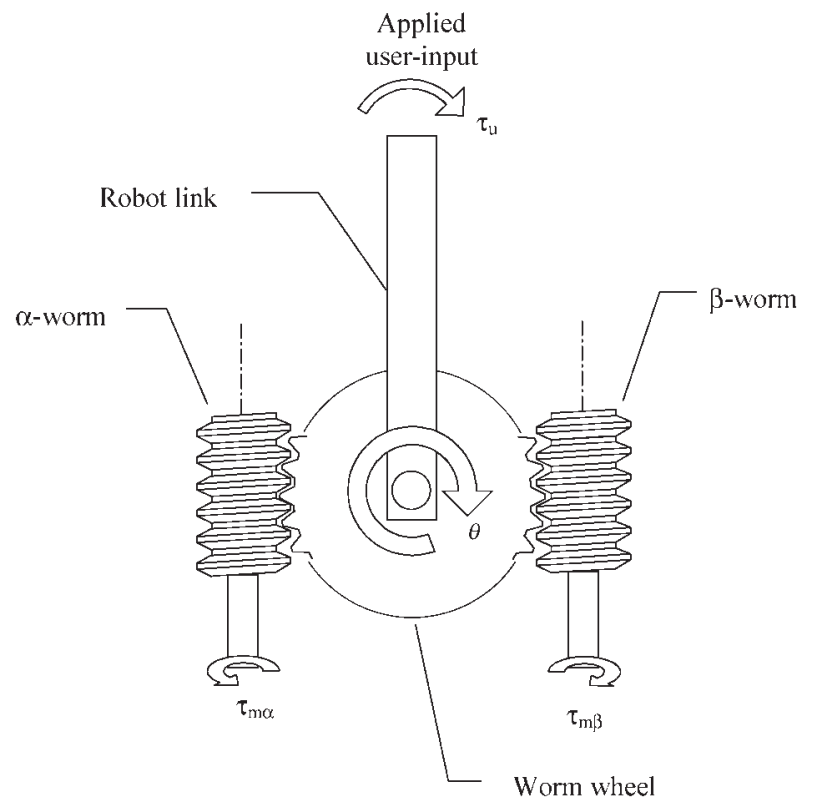

Fig. 1 Dual-worm drive joint 
two worms, each driven by a low-power d.c. servomotor. The two worms follow a worm wheel that is fixed to the robot link. The user provides a force on the end-effector and motion is allowed by controlling the two worms simultaneously. The non-backdriveability of the worm mechanism means that, no matter how much force the operator applies, motion is not allowed until both motors are controlled to move in the same direction.

The parallel nature of this overactuated joint structure makes this active system safer than modified industrial robots. If there were a failure, both motors would have to drive in the same direction for any motion to occur. However, if this failure occurs, acceleration of the joints will be very small owing to the inertia of the links and low power of the motors, giving the operator plenty of time to react, i.e. to release the deadman handle. The dual-worm mechanism also has the ability to eliminate backlash using a control strategy that is discussed below. The control of the joint must be robust against the nonlinear effects of inertia and frictional forces at the worm/ wheel interface.

\section{MATHEMATICAL MODEL}

Analysis of the dual-worm mechanism identifies four separate cases of contact between the two worms and the worm wheel. The first of these four cases (case I), shown in Fig. 2a, represents the worm wheel in contact with the leading edges of the thread of both the $\alpha$-and $\beta$-worms. In this condition the two worms cancel backlash. Figure $2 b$ shows the second case (case II) where the worm wheel is in contact with the trailing edge of the thread of the $\alpha$-worm and the leading edge of the thread of the $\beta$-worm. This situation is highly undesirable in terms of positional accuracy, as backlash is not cancelled. The third case (case III), illustrated in Fig. 2c, shows the trailing edges of the thread of the two worms in contact with the worm wheel. Backlash is cancelled in this condition. Finally, in Fig. 2d (case IV), the leading edge of the thread of the $\alpha$-worm and the trailing edge of the thread of the $\beta$-worm are shown in contact with the wheel. Similar to case II, backlash is not cancelled in this condition. Therefore, backlash is eliminated if either case I or case III is continuously maintained. In order to simplify the model, a control strategy is chosen that will maintain the conditions required for case I only.

Unwinding the thread of the three gears allows the mechanism to be modelled as a system of three wedges sliding against each other. A free-body diagram for case I is shown in Fig. 3, where the upper wedge represents the $\alpha$-worm, the middle wedge represents the worm wheel and the bottom wedge represents the $\beta$-worm. Using Fig.

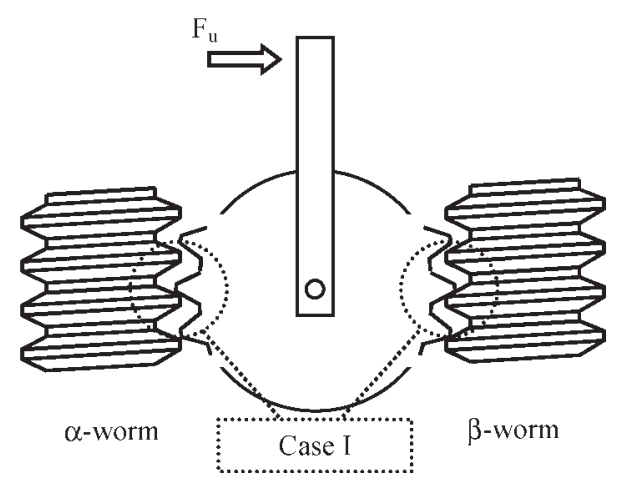

(a)

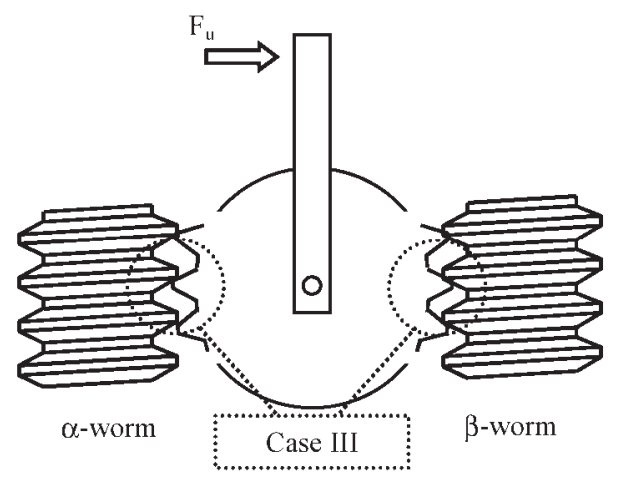

(c)

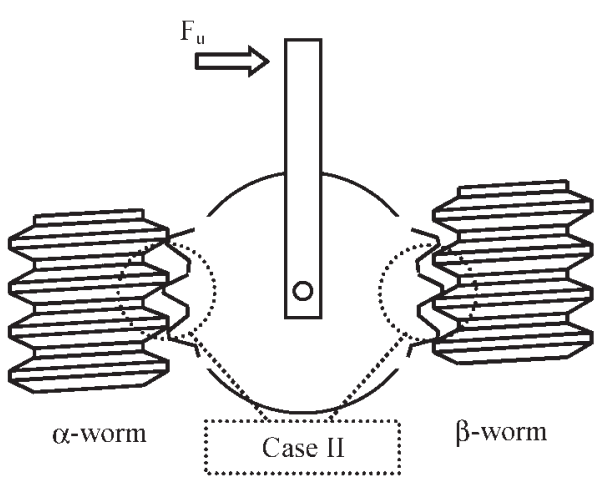

(b)

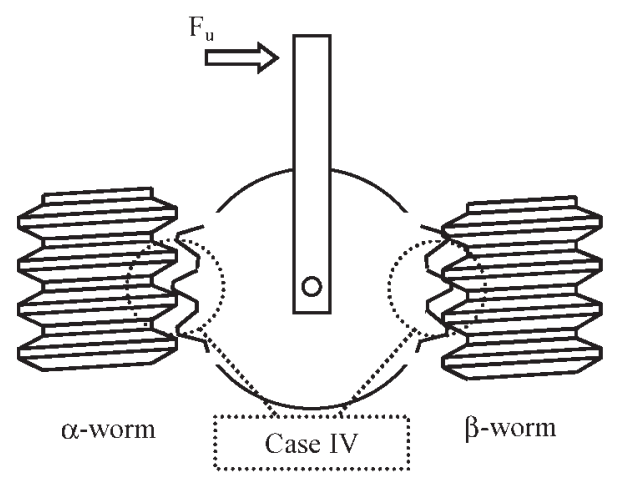

(d)

Fig. 2 Dual-worm joint modes of contact 


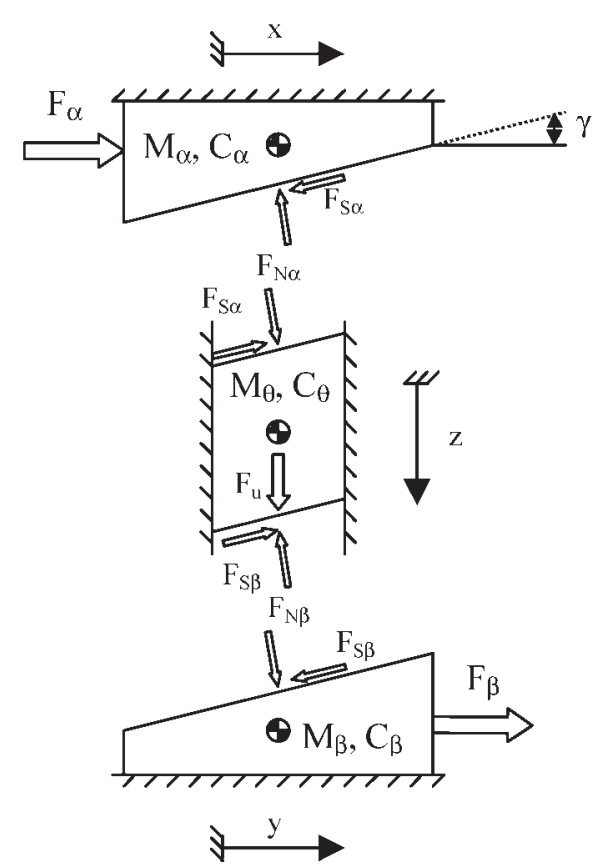

Fig. 3 Free-body diagram of the worm drive joint: case I

3 , it is possible to write the dynamic equations of each wedge as equations (1) to (3) respectively. The friction forces, $F_{\mathrm{S} \alpha}$ and $F_{\mathrm{S} \beta}$, between the two worms and the wheel are modelled using an exponential stick-slip friction model $[9,10]$ given in equations (4) and (5) respectively. In order to simplify the model, it is assumed that neither the $\alpha$-worm nor the $\beta$-worm loses contact with the worm wheel, i.e. $F_{\mathrm{N} \alpha} \neq 0$ and $F_{\mathrm{N} \beta} \neq 0$ :

$$
\begin{aligned}
M_{\alpha} \ddot{x}+C_{\alpha} \dot{x}= & F_{\alpha}-F_{\mathrm{N} \alpha} \sin \gamma-F_{\mathrm{S} \alpha} \cos \gamma \\
M_{\theta} \ddot{z}+C_{\theta} \dot{z}= & F_{\mathrm{u}}+F_{\mathrm{N} \alpha} \cos \gamma-F_{\mathrm{S} \alpha} \sin \gamma-F_{\mathrm{N} \beta} \cos \gamma \\
& +F_{\mathrm{S} \beta} \sin \gamma \\
M_{\beta} \ddot{y}+ & C_{\beta} \dot{y}=F_{\beta}+F_{\mathrm{N} \beta} \sin \gamma-F_{\mathrm{S} \beta} \cos \gamma \\
F_{\mathrm{S} \alpha}= & \left(\mu_{\mathrm{d} \alpha}+\left(\mu_{\mathrm{s} \alpha}-\mu_{\mathrm{d} \alpha}\right) \mathrm{e}^{|\dot{x}| /(\varepsilon \cos \gamma)}\right) \operatorname{sgn}(\dot{x}) F_{\mathrm{N} \alpha} \\
F_{\mathrm{S} \beta}= & \left(\mu_{\mathrm{d} \beta}+\left(\mu_{\mathrm{s} \beta}-\mu_{\mathrm{d} \beta}\right) \mathrm{e}^{|\dot{y}| /(\varepsilon \cos \gamma)}\right) \operatorname{sgn}(\dot{y}) F_{\mathrm{N} \beta}
\end{aligned}
$$

Substitution of (1), (3), (4) and (5) into (2) yields

$$
M_{\theta} \ddot{z}+C_{\theta} \dot{z}=F_{\mathrm{u}}+\frac{F_{\alpha}-M_{\alpha} \ddot{x}-C_{\alpha} \dot{x}}{\Delta_{\alpha}}+\frac{F_{\beta}-M_{\beta} \ddot{y}-C_{\beta} \dot{y}}{\Delta_{\beta}}
$$

where

$$
\begin{aligned}
& \Delta_{\alpha}=\frac{\sin \gamma+\left(\mu_{\mathrm{d} \alpha}+\left(\mu_{\mathrm{s} \alpha}-\mu_{\mathrm{d} \alpha}\right) \mathrm{e}^{|\dot{x}| /(\varepsilon \cos \gamma)}\right) \operatorname{sgn}(\dot{x}) \cos \gamma}{\cos \gamma-\left(\mu_{\mathrm{d} \alpha}+\left(\mu_{\mathrm{s} \alpha}-\mu_{\mathrm{d} \alpha}\right) \mathrm{e}^{|\dot{x}| /(\varepsilon \cos \gamma)}\right) \operatorname{sgn}(\dot{x}) \sin \gamma}, \\
& \Delta_{\beta}=\frac{\sin \gamma-\left(\mu_{\mathrm{d} \beta}+\left(\mu_{\mathrm{s} \beta}-\mu_{\mathrm{d} \beta}\right) \mathrm{e}^{|\dot{y}| /(\varepsilon \cos \gamma)}\right) \operatorname{sgn}(\dot{y}) \cos \gamma}{\cos \gamma+\left(\mu_{\mathrm{d} \beta}+\left(\mu_{\mathrm{s} \beta}-\mu_{\mathrm{d} \beta}\right) \mathrm{e}^{|\dot{y}| /(\varepsilon \cos \gamma)}\right) \operatorname{sgn}(\dot{y}) \sin \gamma}
\end{aligned}
$$

Converting from the unwound threads to the rotational system by replacing $F_{\alpha}, F_{\beta}, F_{\mathrm{u}}, M_{\alpha}, M_{\beta}, M_{\theta}, C_{\alpha}, C_{\beta}, C_{\theta}, z$, $x$ and $y$ in equation (6) with $\tau_{\mathrm{m} \alpha} / r_{\alpha}, \tau_{\mathrm{m} \beta} / r_{\beta}, \tau_{\mathrm{u}} / r_{\theta}, J_{\alpha} / r_{\alpha}^{2}$, $J_{\beta} / r_{\beta}^{2}, J_{\theta} / r_{\theta}^{2}, C_{\mathrm{f} \alpha} / r_{\alpha}^{2}, C_{\mathrm{f} \beta} / r_{\beta}^{2}, C_{\mathrm{f} \theta} / r_{\theta}^{2}, \theta r_{\theta}, \theta r_{\theta} / \tan \gamma$ and $\theta r_{\theta} / \tan \gamma$ respectively yields

$$
J_{\mathrm{T}} \ddot{\theta}+C_{\mathrm{T}} \dot{\theta}=\tau_{\mathrm{u}}+\frac{\tau_{\mathrm{m} \alpha} r_{\theta}}{\Delta_{\alpha} r_{\alpha}}+\frac{\tau_{\mathrm{m} \beta} r_{\theta}}{\Delta_{\beta} r_{\beta}}
$$

where

$$
\begin{aligned}
& J_{\mathrm{T}}=J_{\theta}+\frac{J_{\alpha} r_{\theta}^{2}}{\Delta_{\alpha} r_{\alpha}^{2} \tan \gamma}+\frac{J_{\beta} r_{\theta}^{2}}{\Delta_{\beta} r_{\beta}^{2} \tan \gamma} \\
& C_{\mathrm{T}}=C_{\mathrm{f} \theta}+\frac{C_{\mathrm{f} \alpha} r_{\theta}^{2}}{\Delta_{\alpha} r_{\alpha}^{2} \tan \gamma}+\frac{C_{\mathrm{f} \beta} r_{\theta}^{2}}{\Delta_{\beta} r_{\beta}^{2} \tan \gamma}
\end{aligned}
$$

For simulation purposes, it is necessary to ensure that there is no motion until the motor torque of either the $\alpha$-worm or the $\beta$-worm exceeds the static friction forces. Figure 4a shows how the friction forces act on the three wedges during motion of the wheel in the anticlockwise direction, and Fig. $4 \mathrm{~b}$ shows the orientation of the forces during motion of the wheel in the clockwise direction.

From Fig. 4 it is possible to express the equilibrium conditions in the anticlockwise direction [equation (8)] and clockwise direction [equation (9)]. In Fig. 4, $\mu_{\mathrm{S} \alpha} F_{\mathrm{N} \alpha}$. and $\mu_{\mathrm{S} \beta} F_{\mathrm{N} \beta}$ are substituted for $F_{\mathrm{S} \alpha}$ and $F_{\mathrm{S} \beta}$ respectively:

$$
\begin{aligned}
& F_{\mathrm{u}}+F_{\alpha}\left(\frac{\cos \gamma+\mu_{\mathrm{S} \alpha} \sin \gamma}{\sin \gamma-\mu_{\mathrm{S} \alpha} \cos \gamma}\right)+F_{\beta}\left(\frac{\cos \gamma-\mu_{\mathrm{S} \beta} \sin \gamma}{\sin \gamma+\mu_{\mathrm{S} \beta} \cos \gamma}\right)=0 \\
& F_{\mathrm{u}}+F_{\alpha}\left(\frac{\cos \gamma-\mu_{\mathrm{S} \alpha} \sin \gamma}{\sin \gamma+\mu_{\mathrm{S} \alpha} \cos \gamma}\right)+F_{\beta}\left(\frac{\cos \gamma+\mu_{\mathrm{S} \beta} \sin \gamma}{\sin \gamma-\mu_{\mathrm{S} \beta} \cos \gamma}\right)=0
\end{aligned}
$$

Using equation (8) for the system of unwound threads, motion of the wheel in the anticlockwise direction will occur if the condition given by equation (10) is satisfied. Equation (11) gives this condition for the actual 


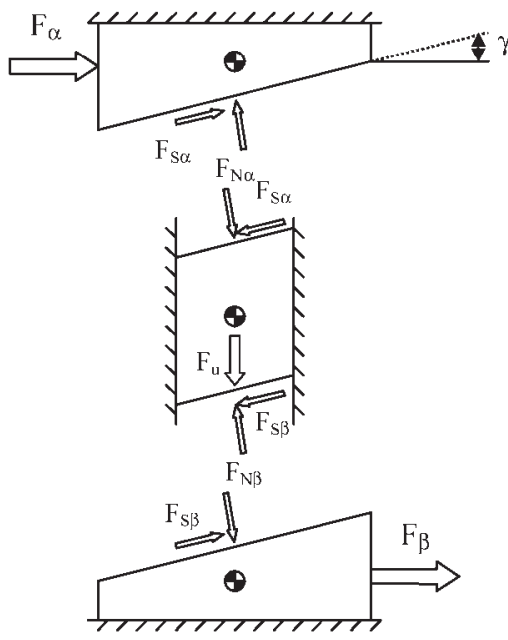

(a) $\alpha$-unwinding

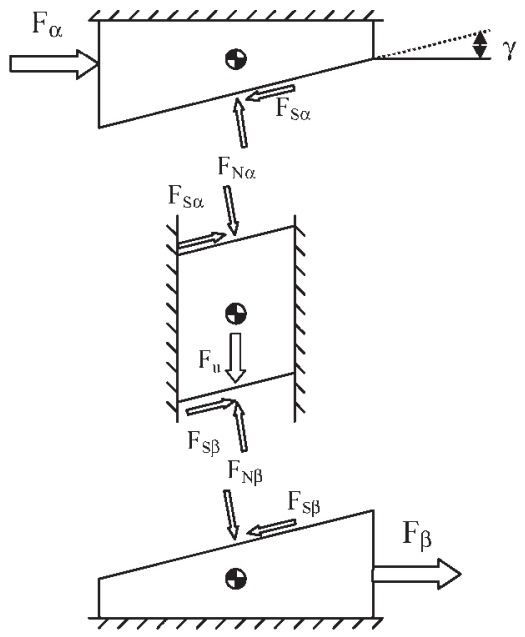

(b) $\beta$-unwinding

Fig. 4 Free-body diagram of static conditions

rotational system:

$$
\begin{gathered}
F_{\alpha}<-F_{\mathrm{u}}\left(\frac{\sin \gamma-\mu_{\mathrm{S} \alpha} \cos \gamma}{\cos \gamma+\mu_{\mathrm{S} \alpha} \sin \gamma}\right)-F_{\beta}\left(\frac{\sin \gamma-\mu_{\mathrm{S} \alpha} \cos \gamma}{\cos \gamma+\mu_{\mathrm{S} \alpha} \sin \gamma}\right) \\
\times\left(\frac{\cos \gamma-\mu_{\mathrm{S} \beta} \sin \gamma}{\sin \gamma+\mu_{\mathrm{S} \beta} \cos \gamma}\right)<0 \quad \text { and } \quad F_{\beta}<0
\end{gathered}
$$

$$
\begin{gathered}
\frac{\tau_{\mathrm{m} \alpha}}{r_{\alpha}}<-\frac{\tau_{\mathrm{u}}}{r_{\theta}}\left(\frac{\sin \gamma-\mu_{\mathrm{S} \alpha} \cos \gamma}{\cos \gamma+\mu_{\mathrm{S} \alpha} \sin \gamma}\right)-\frac{\tau_{\mathrm{m} \beta}}{r_{\beta}}\left(\frac{\sin \gamma-\mu_{\mathrm{S} \alpha} \cos \gamma}{\cos \gamma+\mu_{\mathrm{S} \alpha} \sin \gamma}\right) \\
\times\left(\frac{\cos \gamma-\mu_{\mathrm{S} \beta} \sin \gamma}{\sin \gamma+\mu_{\mathrm{S} \beta} \cos \gamma}\right)<0 \quad \text { and } \quad \tau_{\mathrm{m} \beta}<0
\end{gathered}
$$

Similarly, using equation (9) for the system of unwound threads, clockwise rotation of the wheel will be allowed if the condition given by equation (12) is satisfied. Equation (13) gives the same condition in terms of the actual rotational system:

$$
\begin{gathered}
F_{\beta}>-F_{\mathrm{u}}\left(\frac{\sin \gamma-\mu_{\mathrm{S} \beta} \cos \gamma}{\cos \gamma+\mu_{\mathrm{S} \beta} \sin \gamma}\right)-F_{\alpha}\left(\frac{\sin \gamma-\mu_{\mathrm{S} \beta} \cos \gamma}{\cos \gamma+\mu_{\mathrm{S} \beta} \sin \gamma}\right) \\
\times\left(\frac{\cos \gamma-\mu_{\mathrm{S} \alpha} \sin \gamma}{\sin \gamma+\mu_{\mathrm{S} \alpha} \cos \gamma}\right)>0 \quad \text { and } \quad F_{\alpha}>0
\end{gathered}
$$

$$
\begin{gathered}
\frac{\tau_{\mathrm{m} \beta}}{r_{\beta}}>-\frac{\tau_{\mathrm{u}}}{r_{\theta}}\left(\frac{\sin \gamma-\mu_{\mathrm{S} \beta} \cos \gamma}{\cos \gamma+\mu_{\mathrm{S} \beta} \sin \gamma}\right)-\frac{\tau_{\mathrm{m} \alpha}}{r_{\alpha}}\left(\frac{\sin \gamma-\mu_{\mathrm{S} \beta} \cos \gamma}{\cos \gamma+\mu_{\mathrm{S} \beta} \sin \gamma}\right) \\
\times\left(\frac{\cos \gamma-\mu_{\mathrm{S} \alpha} \sin \gamma}{\sin \gamma+\mu_{\mathrm{S} \alpha} \cos \gamma}\right)>0 \quad \text { and } \quad \tau_{\mathrm{m} \alpha}>0
\end{gathered}
$$

\section{CONTROL DEVELOPMENT}

The aim of the control algorithm is to make the manipulator track a desired position command while cancelling backlash at the worm interface. In order to ensure surgeon and patient safety, the joint and control method must not exhibit:

(a) any motion against the user,

(b) any motion without direct control from the user or

(c) any backlash at the worm/wheel interface.

To this end, effective control of the dual-worm mechanism requires two algorithms. In the clockwise direction (i.e. $\tau_{\mathbf{u}}>0$ ) the motor command voltage for the $\alpha$-worm is set to a constant value and the $\beta$-motor torque, $\tau_{\mathrm{m} \beta}$, is controlled to unwind the $\beta$-worm to track the trajectory. In this condition the $\beta$-worm leads and the $\alpha$-worm is used to follow the motion of the worm wheel without applying unnecessary frictional forces to the system. Control of motion in this manner shall be termed $\beta$-unwinding control. However, in the anticlockwise direction (i.e. $\tau_{\mathbf{u}}<0$ ) the $\beta$-worm motor command 
voltage is set to a constant value and $\tau_{\mathrm{m} \alpha}$ is used to control the unwinding of the $\alpha$-worm to track the trajectory. This is termed $\alpha$-unwinding control. In this instance, the $\alpha$ worm leads and the $\beta$-worm follows. This dual-control strategy is shown to work well.

For the purpose of this preliminary work, the desired velocity is proportional to the user-input torque. That is

$$
\dot{\theta}_{\mathrm{d}}=K_{\mathrm{u}} \tau_{\mathrm{u}}
$$

where $K_{\mathrm{u}}$ could be chosen as a function of position in order to restrict motion. For a manipulator with more than one DOF, this velocity-limiting algorithm would be used to constrain the user to remain along/within a predefined path/region within the robot workspace.

\section{1 $\alpha$-Unwinding control}

The $\alpha$-unwinding control law regulates the unwinding of the $\alpha$-worm to follow a time-varying trajectory. The error between the desired position and the actual position of the system is defined as

$$
e=\theta_{\mathrm{d}}-\theta
$$

From equations (7) and (15) it is possible to derive the tracking error dynamics given by equation (16):

$$
\ddot{e}=\ddot{\theta}_{\mathrm{d}}+\frac{1}{J_{\mathrm{T}}}\left(C_{\mathrm{T}} \dot{\theta}-\tau_{\mathrm{u}}-\frac{\tau_{\mathrm{m} \alpha} r_{\theta}}{\Delta_{\alpha} r_{\alpha}}-\frac{\tau_{\mathrm{m} \beta} r_{\theta}}{\Delta_{\beta} r_{\beta}}\right)
$$

Using feedback linearization, $\tau_{\mathrm{m} \alpha}$ can be chosen to cancel the dynamic effects of friction and the inertial forces; $\tau_{\mathrm{m} \alpha}$ is obtained as given in equation (17), where $\hat{\Delta}_{\alpha}, \hat{\Delta}_{\beta}, \hat{C}_{T}$ and $\hat{J}_{T}$ are the estimates of $\Delta_{\alpha}, \Delta_{\beta}, C_{\mathrm{T}}$ and $J_{\mathrm{T}}$ respectively:

$$
\tau_{\mathrm{m} \alpha}=\frac{r_{\alpha} \hat{\Delta}_{\alpha}}{r_{\theta}}\left(\hat{J}_{\mathrm{T}} u_{\alpha}+\hat{J}_{\mathrm{T}} \ddot{\theta}_{\mathrm{d}}+\hat{C}_{T} \dot{\theta}-\tau_{\mathrm{u}}-\frac{\tau_{\mathrm{m} \beta} r_{\theta}}{r_{\beta} \hat{\Delta}_{\beta}}\right)
$$

In equation (17) the control $u_{\alpha}$ is chosen to drive the error to zero and $\tau_{\mathrm{m} \beta}$ is the measured $\beta$-motor torque. Convergence of the error to zero is achieved using a proportional + integral + derivative (PID) control law:

$$
u_{\alpha}=K_{\mathrm{v} \alpha} \dot{e}+K_{\mathrm{p} \alpha} e+K_{\mathrm{a} \alpha} \int e \mathrm{~d} t
$$

It is important to note that, if the components of equation (17) are not known exactly, the system parameter estimates are erroneous, and without the integral term in equation (18) there may be a non-zero steady state error.

Proc Instn Mech Engrs Vol 215 Part K

\section{$4.2 \beta$-Unwinding control}

A computed-torque controller for unwinding the $\beta$-worm can be found in a similar fashion to that described in Section 4.1. Equation (7) can be used to derive the $\beta$-motor torque as given in equation (19):

$$
\tau_{\mathrm{m} \beta}=\frac{r_{\beta} \hat{\Delta}_{\beta}}{r_{\theta}}\left(\hat{J}_{\mathrm{T}} u_{\beta}+\hat{J}_{\mathrm{T}} \ddot{\theta}_{\mathrm{d}}+\hat{C}_{\mathrm{T}} \dot{\theta}-\tau_{\mathrm{u}}-\frac{\tau_{\mathrm{m} \alpha} r_{\theta}}{r_{\alpha} \hat{\Delta}_{\alpha}}\right)
$$

However, in this case the control $u_{\beta}$ is chosen to drive the error to zero and $\tau_{\mathrm{m} \alpha}$ is the measured $\alpha$-motor torque. Convergence of the error is achieved using the PID controller:

$$
u_{\beta}=K_{\mathrm{v} \beta} \dot{e}+K_{\mathrm{p} \beta} e+K_{\mathrm{a} \beta} \int e \mathrm{~d} t
$$

\section{EXPERIMENTAL SET-UP}

Figure 5 shows the dual-worm driven joint mechanism. The joint is controlled using a Pentium $233 \mathrm{MHz}$ personal computer running the QNX 4.25 real-time operating system. A schematic diagram of the control system is shown in Fig. 6.

The control algorithm requires measurements of motor torque, user-input force, and position. The userinput force is measured using four strain gauges, in a Wheatstone bridge configuration, mounted on a specially designed section of the link. The $3.27 \mathrm{~W}$ geared d.c. motors are capable of producing $0.4 \mathrm{Nm}$ of torque at the output of the gearbox. Closed-loop regulation of the motor output torque is accomplished by measuring the armature current and implementing a digital proportional + integral (PI) controller. All analogue signals are measured and generated using 12 bit analogue-to-digital converters and 12 bit digital-to-analogue converters. The link rotation is recorded using an encoder and appropriate electronics to generate 20000 counts per revolution, giving a resolution of $0.0003142 \mathrm{rad} /$ count (or $0.018 \%$ count). The encoder position is read as a 24 bit number from an HCTL-1100 motion control interface. The frequency of the control loop is set at $600 \mathrm{~Hz}$, and link rotational velocity measurement is obtained in software by using a backward difference algorithm.

\section{RESULTS AND DISCUSSION}

For the model, given by equation (7), and the controllers of equations (17) and (19), the estimated values of the system parameters are given in Table 1 . The values of 


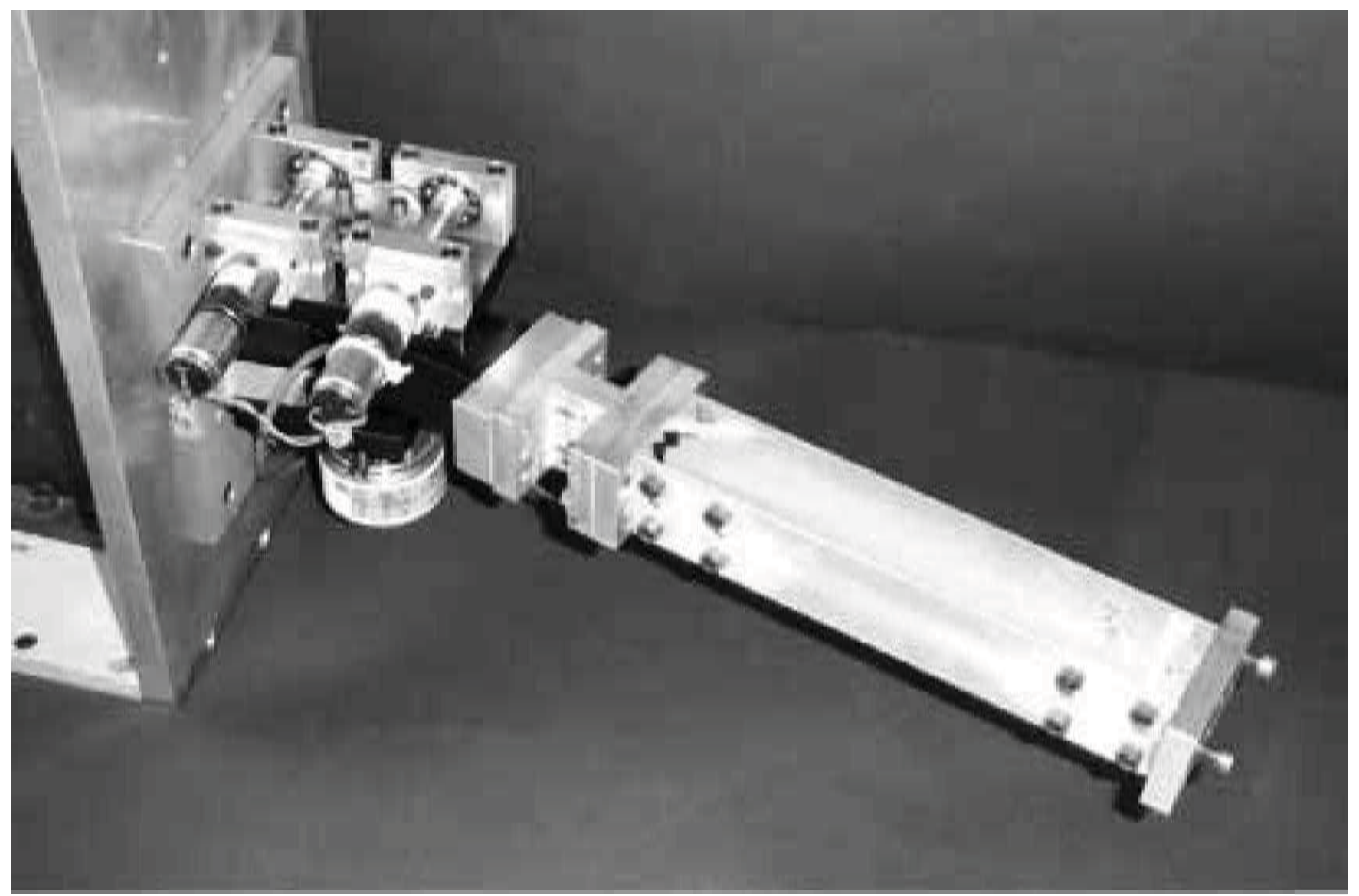

Fig. 5 Worm driven joint

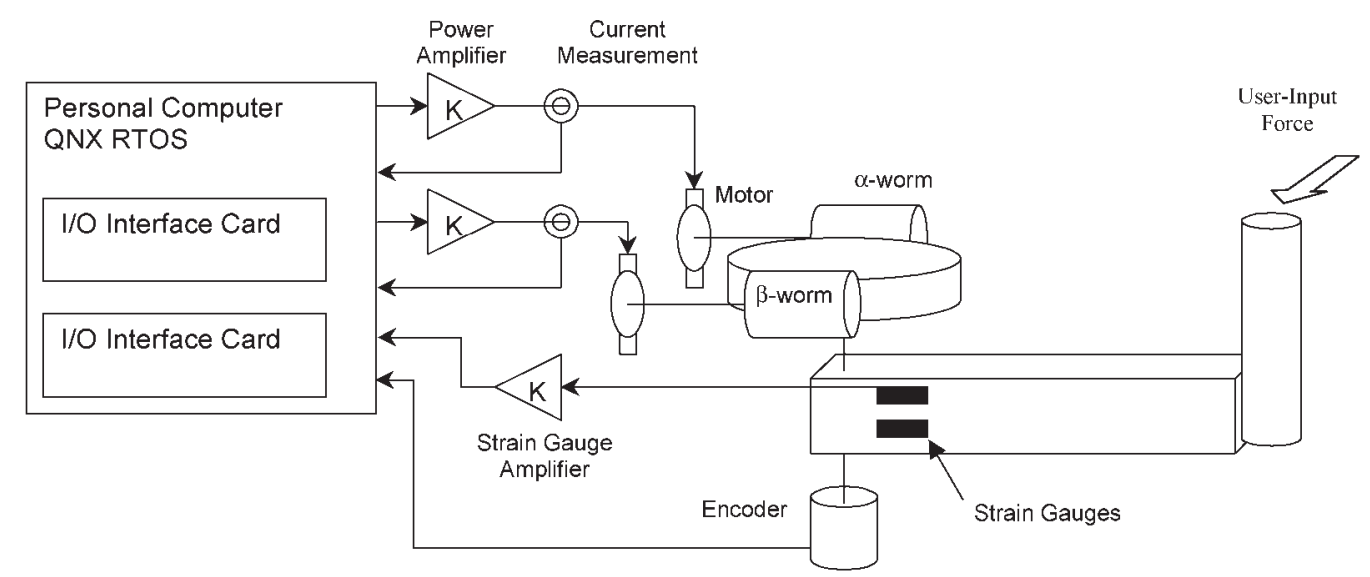

Fig. 6 Schematic overview of the control system

inertia were calculated from the joint geometry. The worm and worm wheel dimensions were assumed to be the pitch centre diameter of the respective gears, while the friction coefficients were determined experimentally. It should be noted that there will be variations in the coefficients of friction owing to temperature and wear.

Table 1 System parameters

\begin{tabular}{llll}
\hline & Worm wheel & $\alpha$-Worm & $\beta$-Worm \\
\hline Inertia $\left(\mathrm{kg} \mathrm{m}^{2}\right)$ & $J_{\theta}=0.062833$ & $J_{\alpha}=0.002855$ & $J_{\beta}=0.002855$ \\
Viscous damping $(\mathrm{N} \mathrm{m} \mathrm{s} / \mathrm{rad})$ & $C_{\mathrm{f} \theta}=0.005$ & $C_{\mathrm{f} \alpha}=0.001$ & $C_{\mathrm{f} \beta}=0.001$ \\
Gear geometry $(\mathrm{m})$ & $r_{\theta}=0.025$ & $r_{\alpha}=0.0065$ & $r_{\beta}=0.0065$ \\
Static friction & & $\mu_{\mathrm{s} \alpha}=0.12$ & $\mu_{\mathrm{s} \beta}=0.16$ \\
Dynamic friction & & $\mu_{\mathrm{d} \alpha}=0.10$ & $\mu_{\mathrm{d} \beta}=0.14$ \\
Friction constant & & $\varepsilon=0.001$ & $\varepsilon=0.001$ \\
Worm lead angle (rad) & & $\gamma=0.05236$ & $\gamma=0.05236$ \\
\hline
\end{tabular}



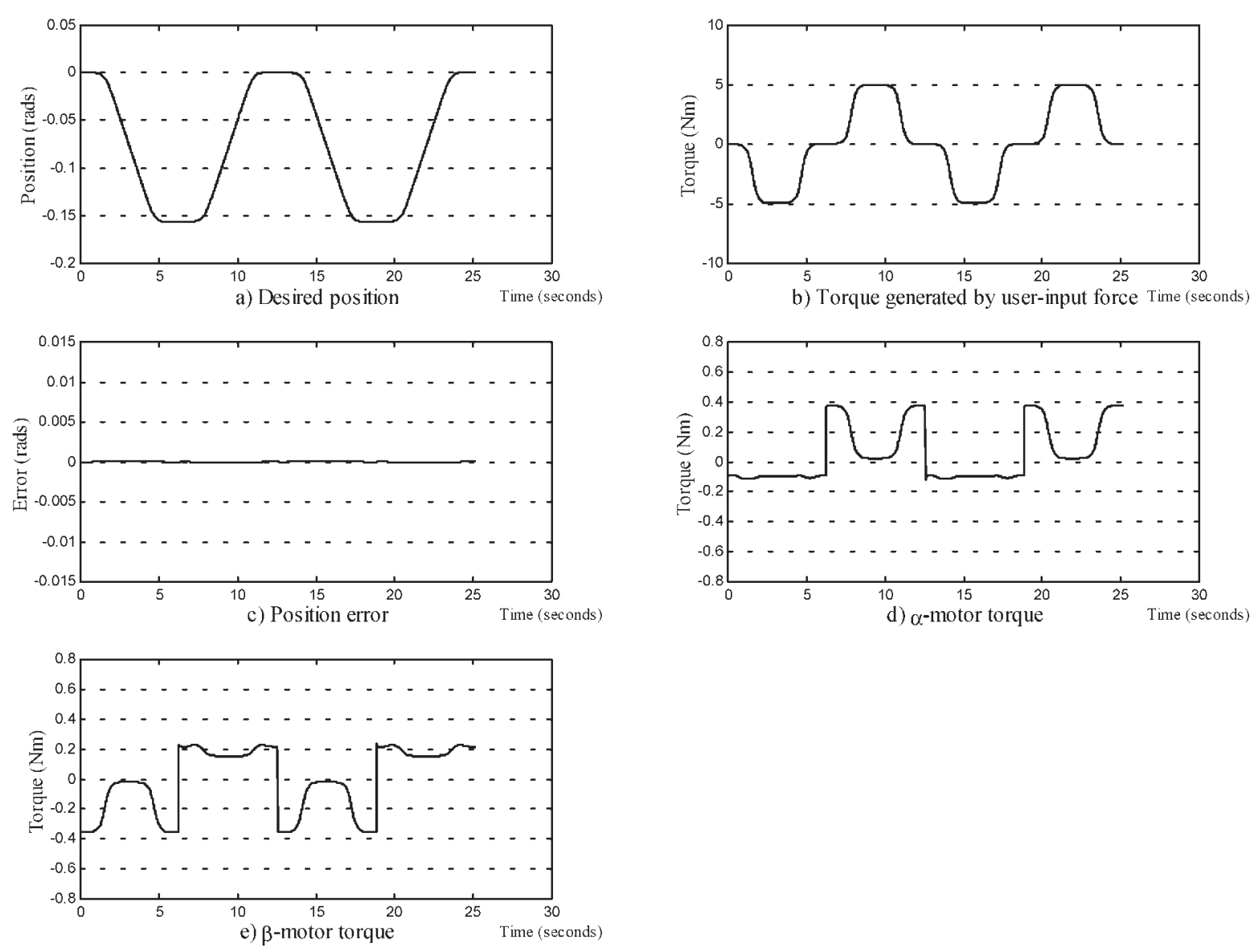

Fig. 7 Dual-worm joint simulation results

These variations may affect the implementation of this system in a multi-DOF robot. Therefore, the robot control system should be calibrated to update the estimates of the friction coefficients periodically, or whenever tracking performance is degraded. This could be done automatically.

\subsection{Simulation results}

In order to test the performance of the control strategy and algorithms, a simulation of the model, given by equation (7), was developed using SIMULINK. Parameters were set according to Table 1, and it was assumed that the control algorithms had exact knowledge of these parameters. Figure 7 shows the results obtained from the simulation. The user input that was applied to the system is given by equation (21) and is shown in Fig. 7b. Figure 7a shows the desired position generated from the speed demand, where $\dot{\theta}_{\mathrm{d}}$ is given by equation (14) with $K_{\mathrm{u}}=$ 0.01. Sufficient tracking performance was achieved using the gains given in Table 2. The graph of position error given in Fig. 7c highlights this. Figures $7 d$ and e show the applied $\alpha$-motor and $\beta$-motor torque used to track the desired trajectory:

$$
\tau_{\mathrm{u}}=2.5 \times \operatorname{sgn}[\sin (0.5 t)]\{\tanh [3 \cos (t)]-1\}
$$

\subsection{Experimental results}

The experimental results for a joint with a single worm are compared with those of the dual-worm mechanism to illustrate backlash cancellation. In both experiments the trajectory is generated by manually applying a force to a control handle attached to the robot link. The velocity demand is generated on the basis of equation (14), where $K_{\mathrm{u}}=0.01$. The desired velocity is then digitally integrated and differentiated to give the desired position and desired acceleration respectively. Figure 8 presents the

Table 2 Simulation gains

\begin{tabular}{llll}
\hline Control direction & Proportional & Integral & Derivative \\
\hline$\alpha$-Unwinding & $K_{\mathrm{p} \alpha}=5000$ & $K_{\mathrm{a} \alpha}=1000$ & $K_{\mathrm{d} \alpha}=100$ \\
$\beta$-Unwinding & $K_{\mathrm{p} \beta}=5000$ & $K_{\mathrm{a} \beta}=1000$ & $K_{\mathrm{d} \beta}=100$ \\
\hline
\end{tabular}



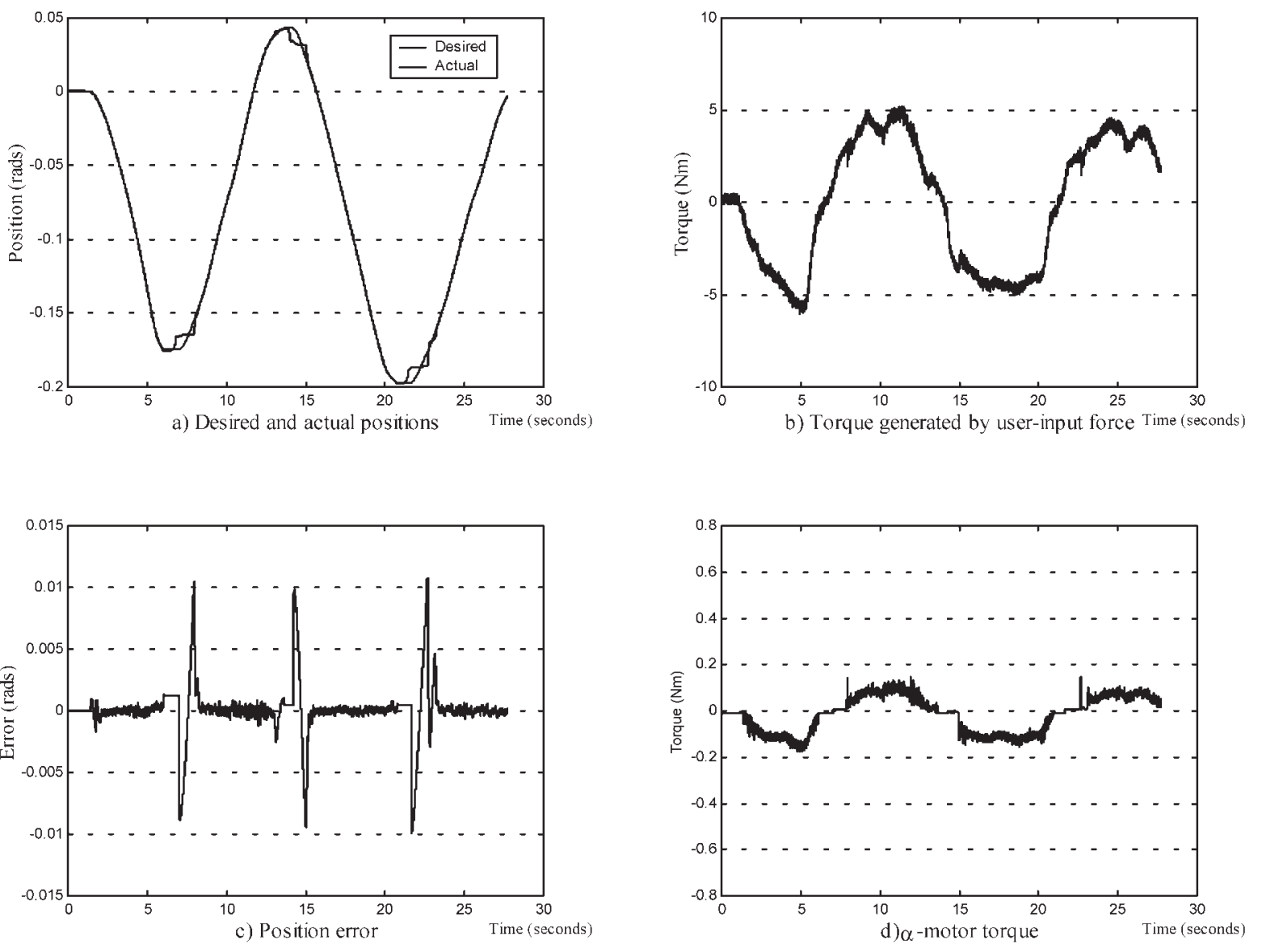

Fig. 8 Single-worm joint experimental results

results for the single-worm mechanism. Figure 8a shows the position demand generated from the user-input force of Fig. 8b. Figure 8c displays the time history of the error during the movement, and Fig. $8 \mathrm{~d}$ illustrates the motor torque used to drive the $\alpha$-worm. It can be seen from Fig. $8 \mathrm{c}$ that the change in direction of the user-input force causes large errors in the order of $\pm 0.01 \mathrm{rad}$ ( or $\pm 0.57^{\circ}$ ). These errors are attributed to the backlash between the worm and worm wheel present in the single-worm system. Figure 9 presents the experimental results of a similar motion to that of the simulation (see Fig. 7) with the dual-worm mechanism. Figure 9a shows the desired and actual positions of the joint. The torque generated by the user-input force is shown in Fig. 9b, and the position error is illustrated in Fig. 9c. It should be noted that the graphs of actual and desired positions in Fig. 9a are indistinguishable because the error (shown in Fig. 9c) is very small. Figures $9 \mathrm{~d}$ and e also show the motor output torque for the $\alpha$-and $\beta$-motors respectively. It is possible to see from Fig. 9c that the backlash in the mechanism has been cancelled and the overall error has been reduced to less than $\pm 0.0015 \mathrm{rad}$ ( or $\pm 0.086^{\circ}$ ).

Tests have also been carried out to determine how the tool-tip position is affected by an external input from the user when the joint is stationary. It has been possible to measure two different sources of error: flexibility of the link and shaft and also end-float of the worm owing to limited preloading of the worm angular contact bearings. Table 3 shows the deflection of the tool tip owing to the two kinds of error for user-input forces of 10 and $20 \mathrm{~N}$ applied at the tool tip. This error was measured at the extremity of the link, a distance $0.4 \mathrm{~m}$ from the centre of rotation. Although this single DOF robot does exhibit some flexibility, $0.4 \mathrm{~mm}$ of error per $10 \mathrm{~N}$ of user-input force is not considered excessive when compared with similar devices. However, increasing the stiffness of the system could further reduce the link and shaft flexibility.

\section{CONCLUSIONS}

In this paper a revolute joint that provides inherent safety features and cancels backlash has been presented. The non-backdriveable dual-worm mechanism is much safer than backdriveable systems in that no motor torque is required to keep the manipulator stationary for most forms of user-input force. However, high-frequency user input (i.e. shaking of the end-effector) can cause the worms to unwind, allowing the mechanism to exhibit 


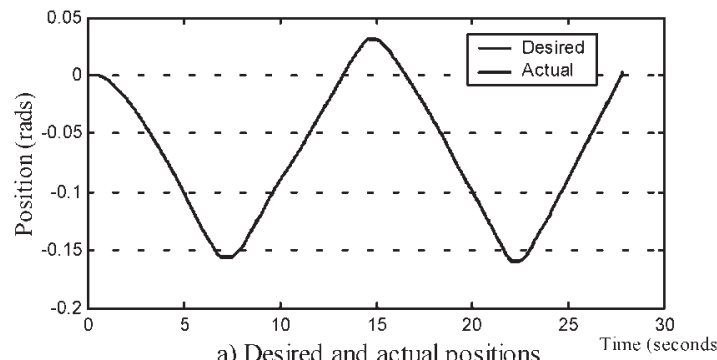

a) Desired and actual positions
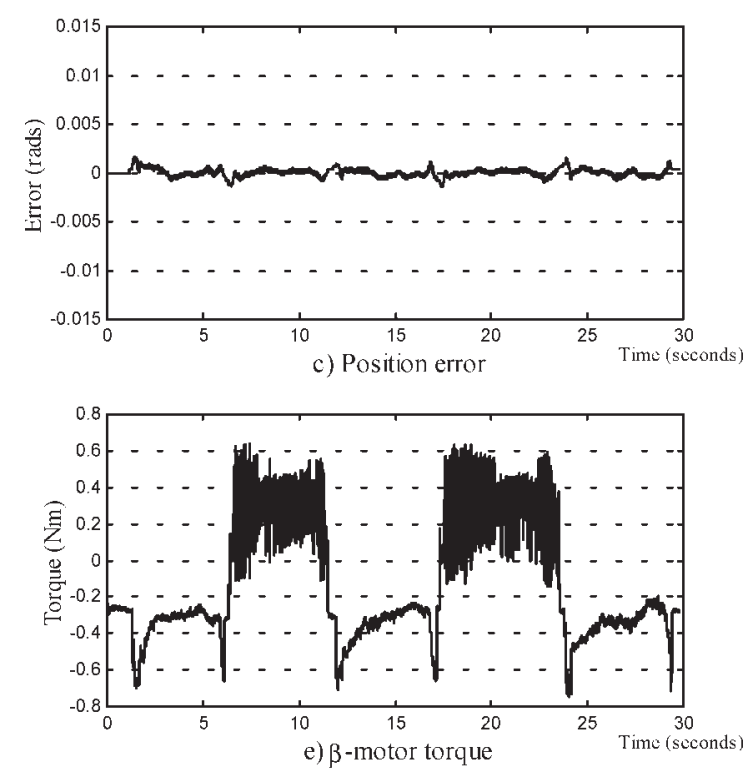

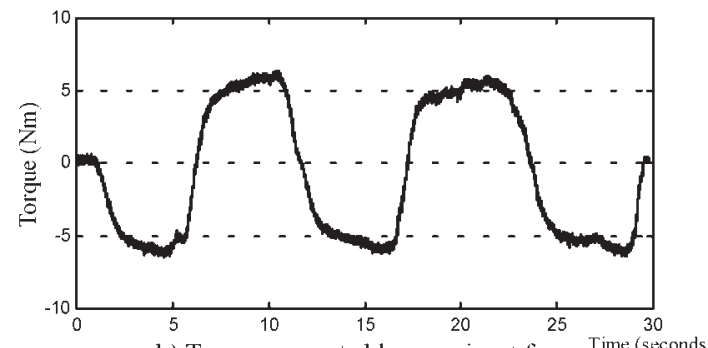

b) Torque generated by user-input force Time (seconds)

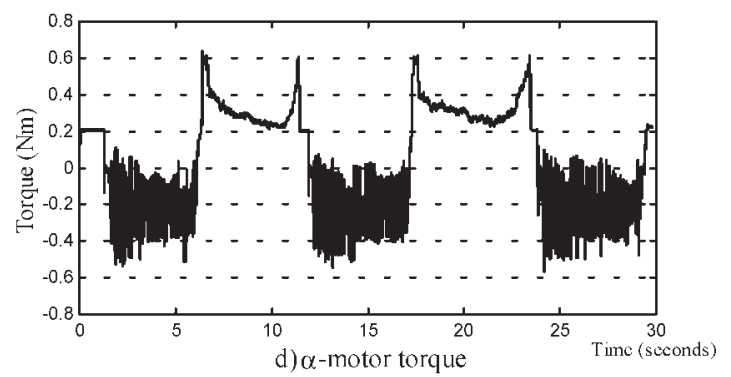

Note: The desired and actual position graphs (Fig. 9a) are indistinguishable because the error ( $\mathrm{Fig} .9 \mathrm{c}$ ) is very small

Fig. 9 Dual-worm joint experimental results

backlash. For this reason, when the joint is stationary, each motor applies a small amount of torque to keep the system backlash free.

Suitable control laws for regulating position have been derived on the basis of a mathematical model of the system. These computed-torque control algorithms have been shown to give good results while tracking a trajectory generated from the measured user-input torque. The control algorithm has been effective in cancelling backlash and compensating for the non-linear effects of friction generated at the interface of the two worms and worm wheel. The mechanical system can also be produced using basic manufacturing technologies and at a low cost when compared with industrial robotic systems.

Table 3 Static error measurements

\begin{tabular}{llll}
\hline & \multicolumn{2}{c}{ Deflection from zero (mm) } \\
\cline { 2 - 4 } $\begin{array}{l}\text { Applied } \\
\text { force }(\mathrm{N})\end{array}$ & Total & $\begin{array}{l}\text { Due to end-float in } \\
\text { worms }\end{array}$ & $\begin{array}{l}\text { Due to link/shaft } \\
\text { flexibility }\end{array}$ \\
\hline 10 & 0.36 & 0.16 & 0.20 \\
20 & 0.74 & 0.32 & 0.42 \\
\hline
\end{tabular}

Proc Instn Mech Engrs Vol 215 Part K

\section{REFERENCES}

1 Taylor, R. H., Mittelstadt, B. D. et al. An image-directed robotic system for precise orthopaedic surgery. IEEE Trans. Robotics and Automn, 1994, 10(3), 261-274.

2 Kazanzides, P., Mittelstadt, B. D. et al. An integrated system for cementless hip replacement. IEEE Engng in Medicine and Biol., 1995, 14(3), 307-313.

3 Davies, B. L. A discussion of safety issues for medical robots. In Computer-Integrated Surgery (Eds R. H. Taylor, S. Lavallée, G. C. Burdea and R. S. Mösges), 1996, pp. 287-296 (MIT Press, Cambridge, Massachusetts).

4 Sainsbury Jr, J. K. The heart of microsurgery. Mech. Engng, December 1998.

5 Harris, S. J., Lin, W. J., Fan, K. L., Hibberd, R. D., Cobb, J. and Davies, B. L. Experiences with robotic systems for knee surgery. Lecture Notes in Computer Sci., 1997, 1205, 757-766.

6 Delnondedieu, Y. and Troccaz, J. PADyC: A Passive Arm with Dynamic Constraints. A prototype with two degrees of freedom. In Proceedings of 2nd Annual International Symposium on Medical Robotics and Computer Assisted Surgery, Baltimore, Maryland, 4-7 November 1995.

7 Troccaz, J. and Delnondedieu, Y. Semi-active guiding systems in surgery. A two-dof prototype of the Passive Arm with Dynamic Constraints (PADyC). Mechatronics, 1996, 6(4), 399-421. 
8 Patton, W. J. Mechanical Power Transmission, 1980 (Prentice-Hall, Englewood Cliffs, New Jersey).

9 Bo, L. C. and Pavelescu, D. The friction-speed relationship and its influence on the critical velocity of stick-slip motion. Wear, 1982, 82, 277-289.
10 Armstrong-Hélouvry, B., Dupont, P. and Canudas De Wit, C. A survey of models, analysis tools and compensation methods for the control of machines with friction. Automatica, 1994, 30(7), 1083-1138. 